\title{
Tendencia de la mortalidad por cáncer en Argentina, Cuba y Uruguay en un período de 15 años*
}

\section{Fifteen-year trends in cancer mortality in Argentina, Cuba and Uruguay}

\author{
Dora Loria'; Juan J. Lence Anta ${ }^{\mathrm{II}}$; Marta E. Guerra Yín ${ }^{\mathrm{III}}$; Yaima Galán \\ Álvarez $^{\mathrm{IV}}$; Enrique Barrios Herrerav; Rafael Alonso Barbeito ${ }^{\mathrm{VI}}$; Graciela \\ Abriata ${ }^{\text {VII }}$; Leticia M. Fernández Garrote \\ IEpidemióloga. Instituto de Oncología "Ángel H. Roffo". Universidad de Buenos \\ Aires. Buenos Aires, Argentina. \\ ${ }^{\mathrm{II}}$ Especialista de II Grado en Bioestadística. Máster en Salud Pública. Investigador \\ Auxiliar. Instituto Nacional de Oncología y Radiobiología. La Habana. Cuba. \\ ${ }^{\mathrm{III}}$ Licenciada en Matemática. Unidad Nacional de Control de Cáncer. Ministerio de \\ Salud Pública. La Habana. Cuba. \\ IV Máster en Salud Pública. Investigador Auxiliar. Unidad Nacional de Control de \\ Cáncer. Ministerio de Salud Pública. La Habana. Cuba \\ ${ }^{\vee}$ Epidemiólogo. Registro Nacional de Cáncer. Comisión Honoraria de Lucha Contra el \\ Cáncer. Montevideo, Uruguay. \\ ${ }^{\mathrm{VI}}$ Licenciado en Matemática. Registro Nacional de Cáncer. Comisión Honoraria de \\ Lucha Contra el Cáncer. Montevideo, Uruguay. \\ ${ }^{\mathrm{VII}}$ Epidemióloga. Dirección de Epidemiología. Ministerio de Salud y Ambiente de la \\ Nación. Buenos Aires, Argentina. \\ VIIIDoctor en Ciencias de la Salud. Especialista de II Grado en Bioestadística. \\ Investigador Titular. Coordinadora del proyecto REDEPICAN. Instituto Nacional de \\ Oncología y Radiobiología. La Habana. Cuba.
}

\section{RESUMEN}

Introducción El cáncer es la segunda causa de muerte en Argentina, Cuba y Uruguay durante la última década, la mortalidad por cáncer ha mostrado una 
tendencia hacia la disminución en los países más desarrollados.

Objetivos Describir la tendencia temporal de la mortalidad por sitios seleccionados de cáncer en Argentina, Cuba y Uruguay durante 1990-2005. Métodos Para cada sitio de cáncer, sexo y país se calcularon las tasas específicas por grupos de edad y ajustadas por el método directo (a la población mundial). Para identificar los puntos de cambio estadísticamente significativos en la tendencia de las tasas ajustadas se utilizó el análisis de regresión lineal segmentada; se estimó el porcentaje de cambio anual.

Resultados Excepto para Cuba, la mortalidad por todas las causas decreció durante el período. Pulmón: se observó una tendencia decreciente en hombres en Argentina y Uruguay (porcentaje estimado de cambio anual: $-2,25$ y $-1,28 \%$ respectivamente), y creciente en mujeres (porcentaje estimado de cambio anual: 1,$75 ; 2,83$ y 3,02 ) en Argentina, Uruguay y Cuba, respectivamente. Mama: tendencia decreciente en los tres países. Próstata: es el segundo tumor en importancia en Cuba y Uruguay, tercero en Argentina. Un cambio negativo en la tendencia se observó desde 1993 (Cuba) y 1998 (Argentina). Colon/recto: tasas decrecientes en Argentina en ambos sexos y estables en Cuba y Uruguay. Esófago: mortalidad decreciente en Argentina y Uruguay. Útero: en los tres países la mortalidad por Útero no especificado fue similar a cuello, disminuyó para Argentina y Uruguay, mientras aumentó en Cuba. El cáncer de cuerpo disminuyó en Argentina.

Conclusiones La mortalidad en Cuba refleja un aumento para los tumores tabacorelacionados. Mayor calidad de la certificación de muerte podría enmascarar una tendencia negativa de la mortalidad por cáncer de cuello de útero. Las tendencias negativas en cáncer de próstata podrían ser resultado de avances en diagnóstico y tratamiento.

Palabras clave: Cáncer, mortalidad, tendencia, regresión lineal segmentada, Argentina, Cuba, Uruguay.

\section{ABSTRACT}

Introduction Cancer is the second cause of death in Argentina, Cuba and Uruguay during the last decade, cancer mortality has shown a decrease trend in developed countries.

Objective To describe mortality trends over time by cancer site in Argentina, Cuba and Uruguay during 1990-2005.

Methods For each cancer site, country and gender, age-group specific and standardised (overall) rates were calculated by direct method (using the world standard population). The jointpoint regression analysis was used to identify the best-fitting points were a statistically significant change in the trend occurred and annual percent change was also estimated.

Results Total cancer mortality rates decline during the whole period excepting for Cuba. Lung: a negative tendency was observed in men in Argentina and Uruguay (annual percent change: -2.25 and $-1.28 \%$ respectively), and increased in women (annual percent change: 1,75, 2,83 and 3,02) in Argentina, Uruguay and Cuba respectively. Breast: Negative trends were observed in the three countries.

Prostate: it is the second cause of mortality in men in Cuba and Uruguay, and third in Argentina. A negative change in the tendency was observed from 1993 (Cuba) and 1998 (Argentina). Colon/recto: Decreasing rates were shown in Argentina in both sexes whereas not changes were noted in Cuba and Uruguay. Esophagus: Negative trends were detected in Argentina and Uruguay. Uterus: in the three countries mortality for Uterus non-specified was similar to cervix, diminishing for Argentina and Uruguay, while increased in Cuba. Body of uterus mortality 
diminished in Argentina.

Conclusions Mortality trends in Cuba indicate an increasing for the tobacco related-cancer. Enhancing quality of death certification could mask a negative tendency of the mortality for cervix cancer. Negative trends in prostate cancer might be view from the impact of advances in diagnosis and treatment.

Key words: Cancer, mortality, trend, jointpoint regression, Argentina, Cuba, Uruguay.

\section{INTRODUCCIÓN}

El incremento de los factores de exposición, muchos de ellos relacionados con factores ambientales unidos a los procesos de transición epidemiológica y demográfica, son algunos de los principales responsables de una mayor carga de morbilidad y mortalidad por cáncer en casi todo el mundo.

El proceso de envejecimiento demográfico observado en muchos países es el resultado del descenso sostenido de la fecundidad y del aumento de las expectativas de vida, los que a su vez se relacionan con factores socioeconómicos. Por su parte, estos procesos son en suma el reflejo de una mayor capacidad de los estados de evitar la muerte temprana y en muchos casos posibilitar que las parejas puedan determinar libremente el número de hijos deseados. Desde esta perspectiva, el envejecimiento constituye un éxito de la salud pública y un mayor ejercicio de derechos. ${ }^{1}$

En América Latina y el Caribe los indicadores demográficos revelan una región que está envejeciendo paulatina e inexorablemente, sin embargo este envejecimiento es heterogéneo, se produce a un ritmo más rápido que el registrado históricamente en los países desarrollados, y ocurre en un contexto caracterizado por una gran pobreza y una persistente y aguda iniquidad social. ${ }^{2}$ Entre estos países, Argentina, Cuba y Uruguay, comparten en orden creciente de importancia, indicadores demográficos de envejecimiento poblacional que los sitúan al nivel de los países desarrollados. Datos de la Organización Mundial de la Salud (OMS) señalan en el 2006 para estos países, una esperanza de vida al nacer entre 74 y 77 años, los adultos de 65 años y más representan entre el 10 y el $13 \%$ de la población total, la tasa global de fecundidad oscila entre 2,4 y 1,5 hijos por mujer, con poblaciones cuyo ritmo de crecimiento natural es de $1 \%$ anual o menor, y con tasas de mortalidad infantil por debajo de 17 fallecidos menores de un año por cada 100000 nacidos vivos. ${ }^{3}$

Durante el período 1990-2005, en Argentina, Cuba y Uruguay, las defunciones por tumores malignos para todas las edades y ambos sexos, constituyeron la segunda causa de muerte en orden de importancia relativa, ${ }^{4-8}$ precedida sólo por las enfermedades del corazón; lo que significó el $21 \%$, $23 \%$ y $26 \%$ de las defunciones totales, respectivamente para el 2005, comparables a las cifras de países desarrollados como los EE.UU.(22 \%) y España (27\%) en igual período ${ }^{9}$ y constituyendo en consecuencia uno de los problemas prioritarios de la salud pública. 
La distribución de la mortalidad por cáncer es también similar en los tres países. Según datos de Globocan 2002, ${ }^{10}$ con ligeras diferencia locales, los cánceres de próstata, colon-recto y pulmón se encuentran entre las cinco primeras causas de muerte por cáncer en hombres, mientras que en mujeres: mama, colon-recto, cuello y cuerpo de útero y pulmón -sólo para el caso de Cuba- se sitúan entre las primeras.

En la última década, la mortalidad por cáncer ha mostrado una tendencia hacia la disminución en los países más desarrollados. ${ }^{11-13}$ Este comportamiento no es extrapolable a otros países ni a períodos anteriores. Un estudio descriptivo sobre las tendencias de la mortalidad por cáncer en 14 localizaciones seleccionadas en 10 países de Latinoamérica y el Caribe y su comparación con Canadá y EE.UU. durante el periodo 1970-2000, ${ }^{14}$ encuentra diferencias entre los países tanto en la mortalidad por los tumores más frecuentes como en su comportamiento. Por ejemplo, la mortalidad por cáncer en las mujeres muestra una tendencia al descenso en Argentina, Chile, Colombia, Costa Rica y Venezuela mientras que presenta un acenso en México y Cuba.

En Argentina, país extenso y con diferencias tanto en el nivel socioeconómico como en el tipo de etnia predominante en las diversas áreas, se han descrito variaciones de la mortalidad por cáncer dentro del mismo país. ${ }^{15}$ Los cambios en la tendencia temporal de las tasas de incidencia y mortalidad son en parte una consecuencia de cambios en los factores de exposición y fundamentalmente de cambios en las medidas de prevención y control de la enfermedad. ${ }^{16}$ El conocimiento de la mortalidad es fundamental para la vigilancia epidemiológica y por tanto para la toma de decisiones en el ámbito de la salud pública. Igualmente, es un instrumento importantísimo para la evaluación en salud, tanto de la cobertura, calidad y capacidad de resolución de los servicios como de los programas, de acciones específicas e incluso de la disponibilidad de tecnologías médicas. ${ }^{17}$

Durante la última década, el modelo de regresión lineal segmentada (jointpoint regression) ha cobrado creciente interés, como herramienta de utilidad para identificar y describir la ocurrencia de cambios en diferentes períodos en el análisis de la tendencia temporal de las tasas de incidencia y mortalidad. ${ }^{18}$

El objetivo de este estudio es proveer -por vez primera- información actualizada y analizar los cambios recientes en la tendencia de la mortalidad por cáncer por todas las causas y según sitios seleccionados en Argentina, Cuba y Uruguay, países miembros de la red REDEPICAN (Red Iberoamericana de Epidemiología y Sistemas de Información en Cáncer) financiado por el Programa Iberoamericano de Ciencia y Tecnología para el Desarrollo (CYTED), durante el período 1990-2005.

\section{MÉTODOS}

Se realizó un estudio descriptivo de la distribución temporal de la mortalidad por cáncer en sus principales localizaciones en los tres países. Los datos de mortalidad por cáncer para todas las causas y según sitios seleccionados, relativos a un período de 16 años (1990-2005), fueron obtenidos de las bases de datos de los registros nacionales de mortalidad de los diferentes países: Argentina [Dirección de Estadísticas e Información de Salud (DEIS) del Ministerio de Salud de la Nación], Cuba (Dirección Nacional de Estadísticas del Ministerio de Salud Pública), Uruguay (Ministerio de Salud Pública y Comisión Honoraria de Lucha contra el Cáncer). 
Para el período de estudio, los datos de mortalidad por cáncer según sexo, referente a las localizaciones más frecuentes: laringe, pulmón, esófago, estómago, colon-recto, vesícula biliar, mama femenina, cuello de útero, cuerpo de útero, útero sin especificar (NOS), ovario, próstata, vejiga, riñón y muertes por cáncer de todas las causas, incluyen la novena y décima revisión de la Clasificación Internacional de Enfermedades (CIE) ${ }^{19}$

La décima revisión de la CIE comenzó a ser usada por las estadísticas de mortalidad en Argentina (1997), Cuba (2001) y Uruguay (2003), por esto, las categorías de tres dígitos de ambas clasificaciones se tomaron como equivalentes, como se muestra en la tabla 1.

Tabla 1. Código de la Clasificación Internacional de Enfermedades (CIE) y equivalencia según las diferentes revisiones

\begin{tabular}{|l|c|c|}
\hline Sitio de cáncer & CIE-9na. ed. & CIE-10ma. ed. \\
\hline Tumor maligno del esófago & 150 & C15 \\
\hline Tumor maligno del estómago & 151 & C16 \\
\hline Tumor maligno del colon y tumor maligno del recto & 153,154 & C18-21 \\
\hline $\begin{array}{l}\text { Tumor maligno de la vesícula biliar y de otras partes y de las } \\
\text { no especificadas de las vías biliares }\end{array}$ & 156 & C23-24 \\
\hline Tumor maligno de la laringe & 161 & C32 \\
\hline Tumor maligno de la tráquea, de los bronquios y del pulmón & 162 & C33-34 \\
\hline Tumor maligno de la mama de la mujer & 174 & C50 \\
\hline Tumor maligno del útero y NOS. & 179 & C55 \\
\hline Tumor maligno del cuello de útero & 180 & C54 \\
\hline Tumor maligno del cuerpo del útero & 182 & C56 \\
\hline Tumor maligno del ovario & 183 & C61 \\
\hline Tumor maligno de la próstata & 189 & C64-68 \\
\hline Tumor maligno de la vejiga urinaria & $140-199$ & C00-C96 \\
\hline $\begin{array}{l}\text { Tumor maligno del riñón, pelvis renal, uréteres y otros de vías } \\
\text { urinarias }\end{array}$ & & \\
\hline Todas las causas & & 188 \\
\hline
\end{tabular}

Para cada año, las poblaciones estimadas a mitad de período fueron tomadas, en Argentina, del Instituto Nacional de Estadística y Censos (INDEC) según las proyecciones intercensales correspondientes a los censos 1991 y 2001. Para Cuba de las estimaciones censales y proyecciones intercensales de población (Censos 1981 y 2002), del Centro de Estudios de Población y Desarrollo de la Oficina Nacional de Estadísticas de (ONE). Los datos de población de Uruguay pertenecen a las estimaciones y proyecciones realizadas por el Instituto Nacional de Estadística (censos de 1985, 1996 y 2004). ${ }^{20}$

Para cada país, por localización y sexo se calcularon las tasas anuales ajustadas por edad (grupos quinquenales) a la población estándar mundial mediante el método de estandarización directo, la tasa ajustada de mortalidad promedio anual para el período 2001-2005, y el intervalo de confianza (aproximación Poisson) de la estimación, con una confiabilidad del $95 \%$ (IC $95 \%$ ). Las tasas fueron expresadas como casos/100 000 años-persona. ${ }^{21,22}$ 
Para el análisis de la tendencia temporal de las tasas ajustadas de mortalidad según localización de cáncer y país, se utilizó el método de regresión lineal segmentada (joinpoint regression). ${ }^{18,23-25}$

Para el cálculo de la regresión lineal segmentada y el porcentaje estimado de cambio anual (PECA) se usó el programa Joinpoint Regression, versión 2, 6 Marzo 2002 de la División de Control de Cáncer y Ciencias de Población del Instituto Nacional del Cáncer de EE.UU. ${ }^{23}$ (Statistical Research 2002) -disponible en Internet y cuya explotación es libre de cargo.

\section{RESULTADOS}

Entre los años 1990 y 2005 las tasas de mortalidad por cáncer de todas las causas, ajustadas por edad a la población mundial (TAEs), mostraron un riesgo de morir por cáncer más alto en los hombres, con diferencias más marcadas en Uruguay (Fig.). Cuba presentó las tasas más bajas en ambos sexos.

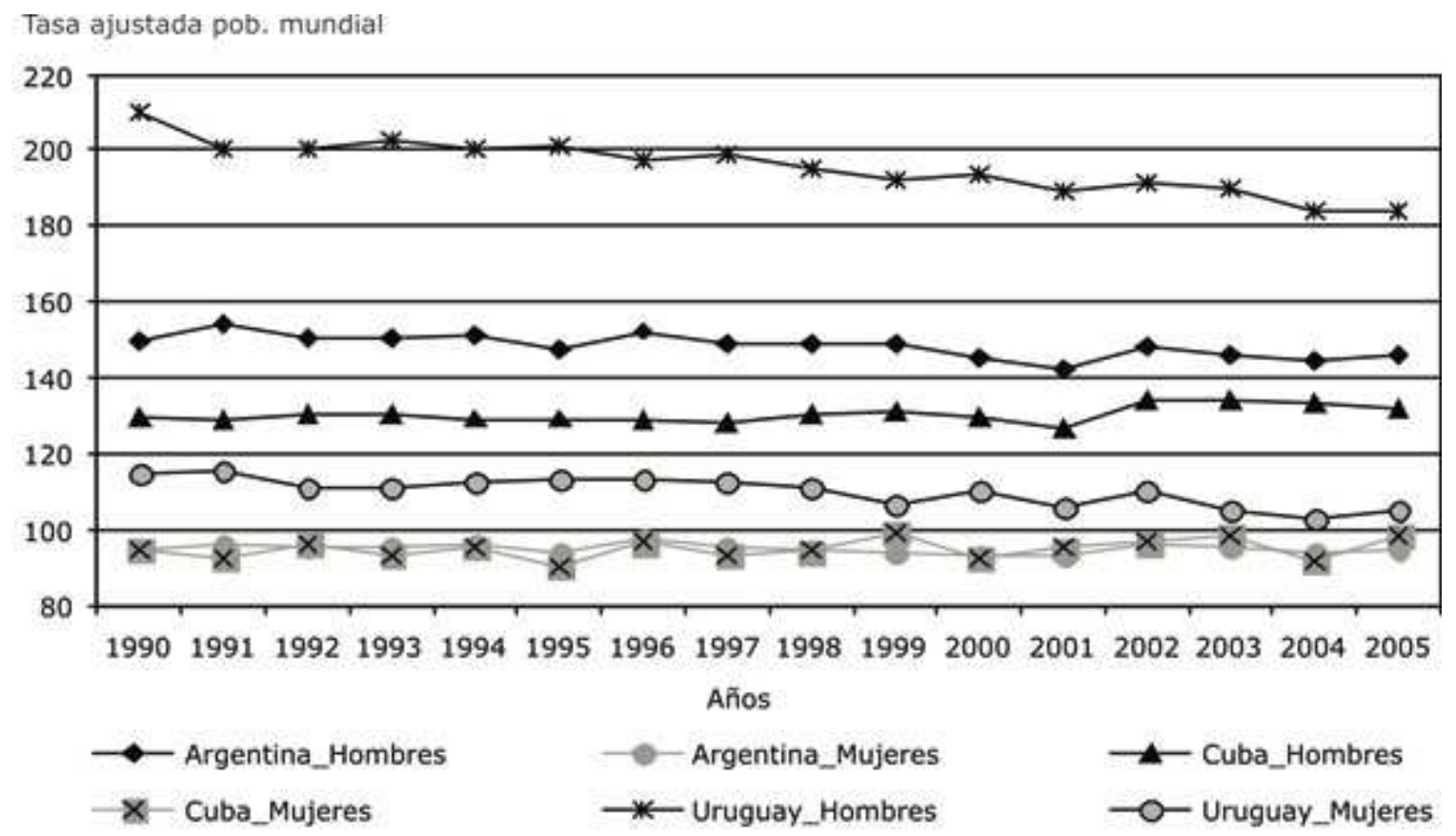

Fig. Tasa de mortalidad por todas las causas de cáncer ajustada por edades a la población estándar mundial.

Durante el período 2001-2005, la tasa estimada promedio anual de mortalidad por cáncer en ambos sexos y para todas las causas, ajustadas por edades a la población mundial, fue significativamente más alta en Uruguay (tabla 2). En los hombres, un patrón similar se observó para casi todos los sitios estudiados, excepto para laringe, pulmón y próstata, con valores significativamente más altos en Cuba y más bajos en Argentina. En el cáncer de las vías biliares, Cuba presentó valores significativamente menores. Para las mujeres, el comportamiento fue diferente (tabla 2). Uruguay exhibió los riesgos de morir significativamente más altos en esófago, estómago, riñón, mama y cáncer del cuello de útero. Cuba mostró un riesgo significativamente mayor de morir por cáncer de laringe, pulmón, cuerpo de útero y útero no especificado (NOS) mientras que este riesgo fue significativamente menor en vías biliares y ovario. La mortalidad en Argentina tuvo valores intermedios, aunque significativamente menores que Cuba y Uruguay, en las localizaciones de colon-recto y vejiga. 
Al comparar las tasas por cáncer de todos los sitios (C00-C80) se observaron - las más bajas- en las mujeres argentinas y para los hombres cubanos.

Tabla 2. Tasa estimada* promedio anual de mortalidad estandarizada e intervalo de confianza (IC)** según sexo y localización. Argentina, Cuba y Uruguay (20012005)

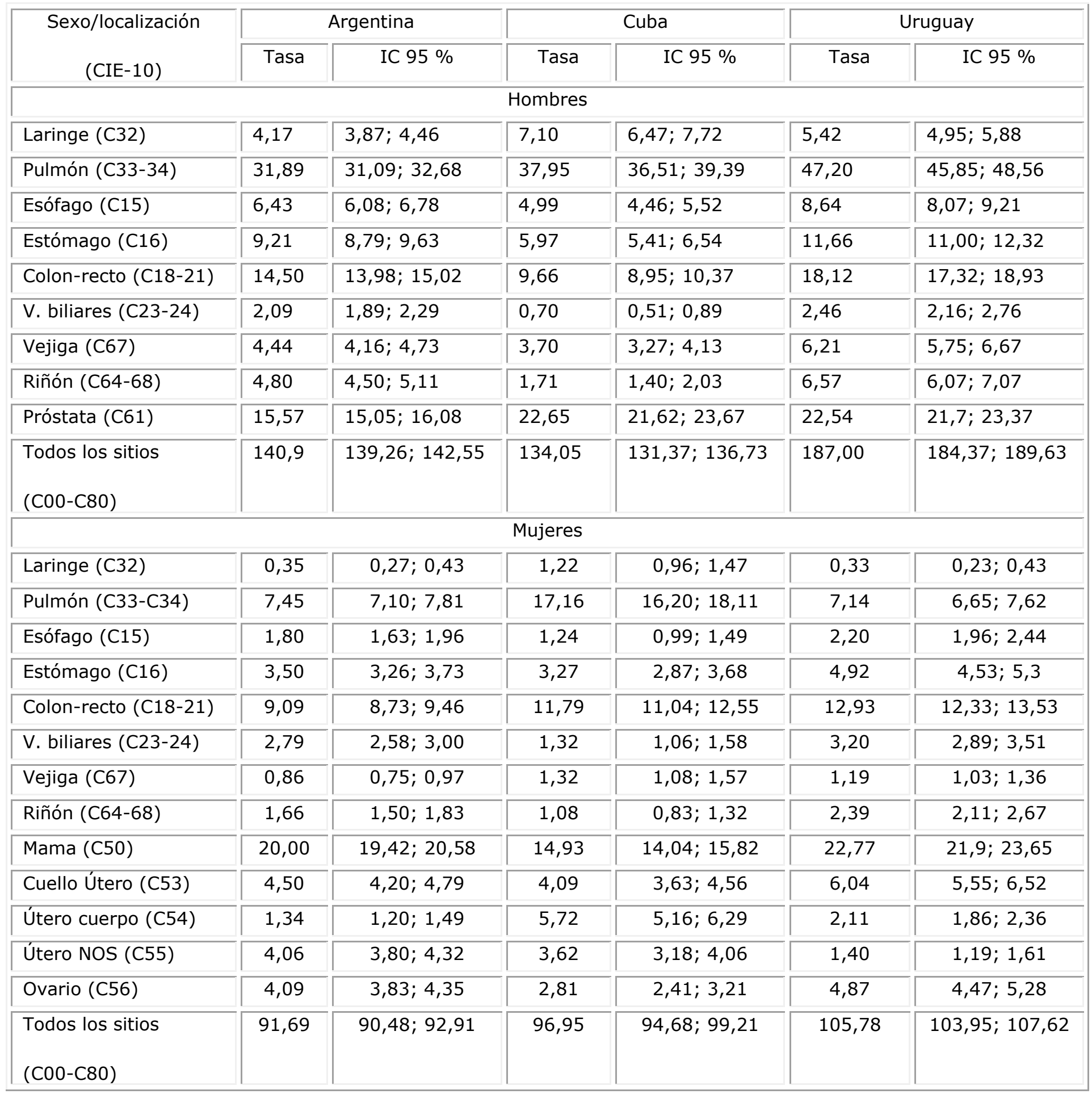

*Tasa por 100000 años-persona; ** IC 95 \% (aproximación Poisson) 
Las tablas 3 y 4 muestran las tendencias de las tasas ajustadas durante el período 1990-2005 y el PECA, por localización y país, en hombres y mujeres, respectivamente. Excepto para Cuba -donde se observaron los valores más bajos-, las TAEs por todas las causas, en el sexo masculino, declinaron significativamente durante 1990 y 2005 a un ritmo de aproximadamente 0,7\% anual para Uruguay y Argentina (figura, tabla 3). Para este último se observó un decremento significativo a partir de finales de la década del 90. Excepto para cáncer de riñón -que mostró un incremento significativo de alrededor de $2 \%$ anual- y próstata -durante los años 90-, las tasas de mortalidad en hombres argentinos decreció significativamente para el resto de las localizaciones durante todo el período, salvo cáncer de vejiga donde se observó un cambio significativo (hacia la estabilidad) en los últimos cuatro años de la serie.

Tabla 3. Tendencia de tasas de mortalidad estandarizadas según país y localización (19902005). Análisis de regresión lineal segmentada. Porcentaje estimado de cambio anual

(PECA). Hombres

\begin{tabular}{|c|c|c|c|c|c|c|c|}
\hline \multirow{2}{*}{$\begin{array}{l}\text { País/localización } \\
\qquad \text { (CIE 10) }\end{array}$} & \multicolumn{2}{|c|}{ Tasa ajustada } & PECA & \multicolumn{2}{|c|}{ Tendencia 1} & \multicolumn{2}{|c|}{ Tendencia 2} \\
\hline & 1990 & 2005 & $1990-05$ & Años & PECA & Años & PECA \\
\hline \multicolumn{8}{|c|}{ Argentina } \\
\hline Laringe (C32) & 5,17 & 4,23 & $-1,72 *$ & $1990-2005$ & $-1,72 *$ & & \\
\hline Pulmón (C33-C34) & 37,30 & 30,37 & $-1,64 *$ & 1990-1996 & $-0,54$ & $1996-2005$ & $-2,25 *$ \\
\hline Esófago (C15) & 8,09 & 6,16 & $-1,99 *$ & $1990-2005$ & $-1,99 *$ & & \\
\hline Estómago (C16) & 11,71 & 9,18 & $-1,76^{*}$ & $1990-2005$ & $-1,76^{*}$ & & \\
\hline Colon-recto (C18-21) & 15,10 & 14,65 & $-0,39 *$ & $1990-2005$ & $-0,39 *$ & & \\
\hline V. biliares (C23-24) & 2,25 & 2,14 & $-1,21 *$ & $1990-2005$ & $-1,21 *$ & & \\
\hline Vejiga (C67) & 5,48 & 4,62 & $-1,90 *$ & $1990-2002$ & $-2,42 *$ & $2002-2005$ & 1,79 \\
\hline Riñón (C64-68) & 3,31 & 4,75 & $2,01^{*}$ & $1990-2005$ & $2,01 *$ & & \\
\hline Próstata (C61) & 12,58 & 15,18 & $1,21 *$ & 1990-1998 & $3,44^{*}$ & $1998-2005$ & $-1,09 *$ \\
\hline $\begin{array}{l}\text { Todos los sitios } \\
(\mathrm{C} 00-\mathrm{C} 80)\end{array}$ & 149,46 & 137,44 & $-0,69 *$ & $1990-1998$ & $-0,19$ & $1998-2005$ & $-1,28 *$ \\
\hline \multicolumn{8}{|l|}{ Cuba } \\
\hline Laringe (C32) & 5,61 & 7,20 & $1,71 *$ & $1990-2005$ & $1,71^{*}$ & & \\
\hline Pulmón (C33-C34) & 38,16 & 37,20 & 0,05 & $1990-1994$ & $-1,86$ & 1994-2005 & 0,51 \\
\hline Esófago (C15) & 4,51 & 5,10 & $0,91 *$ & $1990-2005$ & $0,91 *$ & & \\
\hline Estómago (C16) & 6,68 & 6,20 & $-1,14^{*}$ & $1990-2005$ & $-1,14 *$ & & \\
\hline Colon-recto (C18-21) & 9,88 & 9,51 & $-0,37$ & $1990-2005$ & $-0,37$ & & \\
\hline V. biliares (C23-24) & 1,09 & 0,80 & $-2,76^{*}$ & $1990-2005$ & $-2,76 *$ & & \\
\hline Vejiga (C67) & 4,06 & 3,50 & $-1,06 *$ & $1990-2005$ & $-1,06 *$ & & \\
\hline Riñón (C64-68) & 1,75 & 1,90 & 0,95 & 1992-2005 & 0,95 & & \\
\hline Próstata (C61) & 19,79 & 22,70 & $0,64^{*}$ & $1990-1993$ & $6,10 *$ & 1993-2005 & $-0,08$ \\
\hline $\begin{array}{l}\text { Todos los sitios } \\
(\mathrm{CO0}-\mathrm{C} 80)\end{array}$ & 129,51 & 132,10 & $0,18^{*}$ & $1990-2005$ & $0,18^{*}$ & & \\
\hline \multicolumn{8}{|l|}{ Uruguay } \\
\hline Laringe (C32) & 7,89 & 4,82 & $-2,27 *$ & $1990-2005$ & $-2,27 *$ & & \\
\hline
\end{tabular}


Revista Cubana de Salud Pública; 2010 36(2)115-125

\begin{tabular}{|c|c|c|c|c|c|c|c|}
\hline Pulmón (C33-C34) & 57,02 & 46,31 & $-1,28 *$ & 1990-2005 & $-1,28 *$ & & \\
\hline Esófago (C15) & 10,71 & 7,80 & $-1,78^{*}$ & 1990-2005 & $-1,78^{*}$ & & \\
\hline Estómago (C16) & 16,13 & 10,57 & $-2,49 *$ & 1990-2005 & $-2,49 *$ & & \\
\hline Colon-recto (C18-21) & 17,00 & 17,62 & 0,15 & 1990-2005 & 0,15 & & \\
\hline V. biliares (C23-24) & 2,79 & 2,71 & 0,39 & 1990-2005 & 0,39 & & \\
\hline Próstata (C61) & 21,01 & 21,98 & $0,88^{*}$ & 1990-2005 & $0,88^{*}$ & & \\
\hline $\begin{array}{l}\text { Todos los sitios } \\
(\mathrm{C} 00-\mathrm{C} 80)\end{array}$ & 209,78 & 184,44 & $-0,71^{*}$ & 1990-2005 & $-0,71^{*}$ & & \\
\hline
\end{tabular}

*EI PECA difiere significativamente de cero $(p<0,05) \cdot{ }^{1}$ Datos disponibles solo desde 1992.

Un patrón similar se apreció en la tendencia de la mortalidad en el sexo masculino en Uruguay, con porcentajes de decremento significativamente superiores al $2 \%$ para cáncer de laringe y estómago, un incremento significativo de la mortalidad por cáncer de riñón y próstata, y una tendencia positiva pero no significativa (estable) para cáncer de colon-recto y vejiga.

En Cuba, las localizaciones de estómago, colon-recto, vías biliares y vejiga, en hombres, mostraron una tendencia significativa al decremento entre 1990 y el 2005. Sin embargo, se observó un incremento significativo para el cáncer por todas las causas en todo el período, al igual que para esófago y laringe. Esta última con un incremento de $1,7 \%$ de cambio anual. Para pulmón y próstata, las tendencias de las TAEs a partir de mediados de la década del 90 eran estables.

En el sexo femenino (tabla 4), el análisis de regresión segmentada no mostró para Uruguay cambios de dirección en la tendencia. Sin embargo, para todo el período se observó una tendencia significativamente decreciente de las TAEs para cáncer de esófago, estómago y vías biliares, mama, útero NOS, y cáncer por todas las causas. Tendencias significativamente crecientes fueron observadas para cáncer de pulmón (2,8\% de incremento anual), vejiga y cuello de útero (CCU). Mientras que para cáncer de laringe, riñón, ovario y cuerpo de útero no se detectaron cambios significativos.

Las TAEs por cáncer total en mujeres argentinas mostraron una tendencia significativamente decreciente entre 1996 y el 2005. Se observaron patrones de decremento similares a Uruguay para cáncer de esófago, estómago y vías biliares, aunque en este país disminuyó también el cáncer de colon y recto en las mujeres. Las TAEs de cáncer de mama, cuerpo de útero y útero NOS -este último con un cambio más marcado durante los últimos cinco años-, declinaron significativamente para todo el período. Se observó estabilidad de las pendientes en cáncer de ovario, vejiga y laringe. Un aumento significativo en la pendiente de las TAEs fue estimado para las localizaciones de pulmón ( $1,75 \%$ de cambio anual) y riñón (PECA de $1,08 \%)$.

En Cuba, las TAEs de cáncer de todas las causas en mujeres no mostró cambios significativos durante todo el período. Una declinación importante cercana al $5 \%$ anual para todo el período se apreció para el cáncer de vías biliares, y con un porcentaje menor pero significativamente decreciente para cáncer de estómago. La 
mortalidad por cáncer de pulmón se incrementó significativamente a un ritmo de $3,02 \%$ anual durante 1995-2002. Se observó también un incremento de las tasas de mortalidad por cáncer de riñón (1,83 \% anual) y cuerpo de útero, aunque este incremento fue significativamente más alto (7,94\% anual) durante el período 1992-1998. Para el resto de las localizaciones no se observaron cambios significativos. 
Tabla 4. Tendencia de tasas de mortalidad estandarizadas según país y localización (19902005). Análisis de regresión lineal segmentada. Porcentaje estimado de cambio anual (PECA). Mujeres

\begin{tabular}{|c|c|c|c|c|c|c|c|}
\hline \multirow{2}{*}{$\begin{array}{l}\text { País/Localización } \\
\text { (CIE 10) }\end{array}$} & \multicolumn{2}{|c|}{ Tasa ajustada } & \multirow{2}{*}{$\begin{array}{l}\text { PECA } \\
1990- \\
05\end{array}$} & \multicolumn{2}{|c|}{ Tendencia 1} & \multicolumn{2}{|c|}{ Tendencia 2} \\
\hline & 1990 & 2005 & & Años & PECA & Años & PECA \\
\hline \multicolumn{8}{|c|}{ Argentina } \\
\hline Laringe (C32) & 0,33 & 0,41 & 0,37 & $1990-2005$ & 0,37 & & \\
\hline $\begin{array}{l}\text { Pulmón (C33- } \\
\text { C34) }\end{array}$ & 5,78 & 7,73 & $1,75^{*}$ & $1990-2005$ & $1,75^{*}$ & & \\
\hline Esófago (C15) & 2,10 & 1,73 & $-1,37 *$ & $1990-2005$ & $-1,37 *$ & & \\
\hline Estómago (C16) & 4,63 & 3,48 & $-2,37^{*}$ & $1990-2005$ & $-2,37^{*}$ & & \\
\hline $\begin{array}{l}\text { Colon-recto } \\
(\mathrm{C} 18-21)\end{array}$ & 10,30 & 9,33 & $-0,93 *$ & $1990-2005$ & $-0,93 *$ & & \\
\hline $\begin{array}{l}\text { V. biliares (C23- } \\
24)\end{array}$ & 3,51 & 2,80 & $-1,97^{*}$ & $1990-2005$ & $-1,97^{*}$ & & \\
\hline Vejiga (C67) & 1,00 & 0,82 & $-0,78$ & $1990-2005$ & $-0,78$ & & \\
\hline Riñón (C64-68) & 1,43 & 1,71 & $1,08^{*}$ & 1990-2005 & $1,08^{*}$ & & \\
\hline Mama (C50) & 20,56 & 19,51 & $-0,43 *$ & $1990-2005$ & $-0,43 *$ & & \\
\hline $\begin{array}{l}\text { Cuello Útero } \\
\text { (C53) }\end{array}$ & 4,18 & 4,06 & $-0,25$ & $1990-2005$ & $-0,25$ & & \\
\hline $\begin{array}{l}\text { Útero cuerpo } \\
\text { (C54) }\end{array}$ & 2,07 & 1,26 & $-2,94 *$ & 1990-2005 & $-2,94 *$ & & \\
\hline $\begin{array}{l}\text { Útero NOS } \\
(\text { C55) }\end{array}$ & 4,52 & 3,80 & $-0,81^{*}$ & $1990-2000$ & 0,62 & $\begin{array}{l}2000- \\
2005\end{array}$ & $-4,57 *$ \\
\hline Ovario (C56) & 3,78 & 4,04 & 0,13 & 1990-2005 & 0,13 & & \\
\hline $\begin{array}{l}\text { Todos los sitios } \\
(\mathrm{C} 00-\mathrm{C} 80)\end{array}$ & 94,70 & 94,97 & $-0,07^{*}$ & $1990-2005$ & $-0,07^{*}$ & & \\
\hline \multicolumn{8}{|c|}{ Cuba } \\
\hline Laringe (C32) & 1,36 & 1,4 & 0,76 & $1990-2005$ & 0,76 & & \\
\hline $\begin{array}{l}\text { Pulmón (C33- } \\
\text { C34) }\end{array}$ & 14,0 & 18,7 & $1,91^{*}$ & 1990-1995 & $-1,01$ & $\begin{array}{l}1995- \\
2005\end{array}$ & $3,02 *$ \\
\hline Esófago (C15) & 1,41 & 1,1 & $-0,78$ & $1990-2001$ & -0.54 & $\begin{array}{l}2001- \\
2005\end{array}$ & -6.17 \\
\hline Estómago (C16) & 3,68 & 3,1 & $-0,96 *$ & 1990-2005 & $-0,96 *$ & & \\
\hline $\begin{array}{l}\text { Colon-recto } \\
(\mathrm{C} 18-21)\end{array}$ & 11,44 & 12,14 & 0,02 & 1990-2005 & 0,02 & & \\
\hline $\begin{array}{l}\text { V. biliares (C23- } \\
24)\end{array}$ & 2,06 & 0,9 & $-4,60 *$ & 1990-2002 & $-3,02 *$ & $\begin{array}{l}2002- \\
2005\end{array}$ & $-15,37$ \\
\hline Vejiga (C67) & 1,17 & 1,3 & 0,48 & $1990-2005$ & 0,48 & & \\
\hline Riñón (C64-68) & 1,06 & 1,2 & $1.83^{*}$ & $1992-2005$ & $1.83^{*}$ & & \\
\hline Mama (C50) & 14,7 & 14,9 & $-0,32$ & $1990-2005$ & $-0,32$ & & \\
\hline $\begin{array}{l}\text { Cuello Útero } \\
\text { (C53) }\end{array}$ & 5,3 & 5,3 & 0,30 & $1990-2005$ & 0,30 & & \\
\hline Útero cuerpo & 2,95 & 3,6 & $2,30 *$ & 1992-1998 & $7,94 *$ & 1998- & $-2,03$ \\
\hline
\end{tabular}


Revista Cubana de Salud Pública; 2010 36(2)115-125

\begin{tabular}{|c|c|c|c|c|c|c|c|}
\hline (C54) & & & & & & 2005 & \\
\hline $\begin{array}{l}\text { Útero NOS } \\
(\mathrm{C} 55)\end{array}$ & 5,03 & 5,1 & $-2,50$ & $1992-2005$ & $-2,50$ & & \\
\hline Ovario (C56) & 3,32 & 2,9 & $-0,56$ & $1990-2005$ & $-0,56$ & & \\
\hline $\begin{array}{l}\text { Todos los sitios } \\
\text { (C00-C80) }\end{array}$ & 94,79 & 98,4 & 0,18 & $1990-2005$ & 0,18 & & \\
\hline \multicolumn{8}{|c|}{ Uruguay } \\
\hline Laringe (C32) & 0,16 & 0,40 & $-1,55$ & $1990-2005$ & $-1,55$ & & \\
\hline $\begin{array}{l}\text { Pulmón (C33- } \\
\text { C34) }\end{array}$ & 4,56 & 6,92 & $2,83^{*}$ & $1990-2005$ & $2,83^{*}$ & & \\
\hline Esófago (C15) & 2,99 & 2,43 & $-1,62 *$ & $1990-2005$ & $-1,62 *$ & & \\
\hline Estómago (C16) & 6,09 & 4,59 & $-2,74^{*}$ & $1990-2005$ & $-2,74 *$ & & \\
\hline $\begin{array}{l}\text { Colon-recto } \\
(\mathrm{C} 18-21)\end{array}$ & 13,51 & 11,40 & $-0,48$ & $1990-2005$ & $-0,48$ & & \\
\hline $\begin{array}{l}\text { V. biliares (C23- } \\
24)\end{array}$ & 4,15 & 3,14 & $-1,18^{*}$ & $1990-2005$ & $-1,18^{*}$ & & \\
\hline Vejiga (C67) & 1,45 & 2,55 & $1,86^{*}$ & $1990-2005$ & $1,86^{*}$ & & \\
\hline Riñón (C64-68) & 1,40 & 1,57 & $-0,69$ & $1990-2005$ & $-0,69$ & & \\
\hline Mama (C50) & 25,91 & 23,18 & $-0,90 *$ & $1990-2005$ & $-0,90 *$ & & \\
\hline $\begin{array}{l}\text { Cuello Útero } \\
\text { (C53) }\end{array}$ & 5,29 & 5,84 & $1,05^{*}$ & $1990-2005$ & $1,05^{*}$ & & \\
\hline $\begin{array}{l}\text { Útero cuerpo } \\
\text { (C54) }\end{array}$ & 1,94 & 1,55 & 0,19 & $1990-2005$ & 0,19 & & \\
\hline $\begin{array}{l}\text { Útero NOS } \\
(\text { C55) }\end{array}$ & 4,48 & 2,19 & $-7,70 *$ & 1990-2005 & $-7,70 *$ & & \\
\hline Ovario (C56) & 3,63 & 5,49 & 1,00 & 1990-2005 & 1,00 & & \\
\hline $\begin{array}{l}\text { Todos los sitios } \\
(\mathrm{COO}-\mathrm{C} 80)\end{array}$ & 115,1 & 105,2 & $-0,61^{*}$ & $1990-2005$ & $-0,61^{*}$ & & \\
\hline
\end{tabular}

*EI PECA difiere significativamente de cero $(p<0,05) \cdot{ }^{1}$ Datos disponibles solo desde 1992. 


\section{DISCUSIÓN}

Las tres primeras causas de morir por cáncer en los hombres de los países estudiados son pulmón, próstata y colon-recto. El riesgo de morir por cáncer de próstata es casi igual en Uruguay y en Cuba, país que mantiene las tasas más bajas de mortalidad entre los tres. Mientras, Argentina y Uruguay conservan las semejanzas en el cuarto y quinto lugar (estómago y esófago), en Cuba es el cáncer de laringe quien ocupa el cuarto lugar en la mortalidad. Para las mujeres, a pesar de tener un patrón bastante similar, se observan ciertas diferencias ya que Cuba tiene riesgos más altos de morir por cáncer del pulmón y TAEs un poco más bajas en cáncer de cuello de útero.

El cáncer de próstata es uno de los cánceres más frecuentes en el mundo y en numerosos países se ha observado una tendencia descendente en sus tasas de mortalidad, tal como se presenta en este estudio, en Argentina (descenso marcado) y en Cuba (descenso discreto pero significativo). Algunos autores señalan que este comportamiento pudiera ser el resultado de mejoras a nivel del diagnóstico en estadios tempranos y en el tratamiento -combinado con el pesquisaje- mediante el uso del antígeno prostático específico. En Cuba, donde el pesquisaje no ha ocurrido, se observó en los primeros años, una tendencia creciente de la mortalidad debido principalmente al diagnóstico tardío. ${ }^{27}$ Esta situación provocó que se diseñaran estrategias a nivel nacional para el abordaje de este problema y como consecuencia se observa una disminución en el último período. Los resultados de un estudio de factores de riesgo en esta enfermedad realizado en Cuba apoyan la hipótesis de que un factor infeccioso relacionado con los hábitos de vida sexual podría estar implicado en su etiología; así mismo, no se descarta el papel de factores hormonales relacionados con la actividad sexual propiamente dicha o interactuando con enfermedades de transmisión sexual. ${ }^{28}$

Para Sudamérica existen estimaciones de las tasas de mortalidad por cáncer de laringe, ajustadas por edad, de 3,0 para los hombres y 0,6 para las mujeres con variaciones considerables entre países. ${ }^{10}$ En Argentina y Uruguay la mortalidad por cáncer de laringe en hombres disminuyó gradualmente a lo largo del periodo, aunque el PECA fue mayor en Uruguay. Contrariamente, en Cuba la mortalidad por cáncer de laringe mostró las tasas más altas en hombres y un aumento significativo, lo que es consistente con el estudio de Bossetti. ${ }^{14} \mathrm{El}$ tabaco y el alcohol son los factores más relacionados con el cáncer de laringe y en un estudio reciente desarrollado en colaboración con la IARC, ${ }^{29}$ los odd ratio (OR) para ambos factores, en el estudio cubano fueron de 27,2 para los que consumían 30 o más cigarrillos por día y 6,16 para aquellos que bebían 70 o más tragos de alcohol por semana. Existen dudas en cuanto a la razón de la disminución observada en Uruguay ya que no se corresponde, como en el caso de Argentina con una disminución en el cáncer del pulmón. Pudiera pensarse entonces en una mejoría en el diagnóstico, profesionales más preparados o un mejor acceso a los tratamientos por parte de la población, tal como se refleja en un estudio desarrollado en Chile, ${ }^{30}$ aproximadamente durante la misma etapa. Las tasas de mortalidad por cáncer de laringe en las mujeres, no mostraron cambios significativos en el periodo, no obstante debe destacarse que en Cuba la mortalidad es aproximadamente 10 veces mayor que en Uruguay y Argentina, lo que es consistente con la posición que ocupa el cáncer del pulmón en las mujeres en este país y que puede seguramente estar asociado con el hábito de fumar.

Las tasas globales de mortalidad por cáncer de esófago fueron, en ambos sexos, mayores en Uruguay, seguidas por las de Argentina y Cuba. Excepto para los hombres en Cuba, la mortalidad por cáncer de esófago mostró tendencias decrecientes. 
Un estudio publicado recientemente sobre las tendencias de la incidencia y mortalidad por cáncer de esófago en Europa ${ }^{31}$ y en el que se presentan también las curvas temporales de consumo de alcohol y cigarrillos de las poblaciones, muestra para la mortalidad un comportamiento heterogéneo y no siempre ligada a los cambios en el consumo de cigarrillos. También para otros países de América Latina se encuentran disminuciones en las tasas de mortalidad por esta causa. ${ }^{32}$ Como ocurre con el cáncer de laringe, un bajo consumo de frutas y vegetales está relacionado con el cáncer de esófago, supuestos que para Cuba se presentan de forma contradictoria.

Los tumores malignos del pulmón ocuparon el primer lugar en la mortalidad por cáncer para los hombres de los tres países y las mujeres en Cuba. Una mortalidad mayor por cáncer de pulmón en los hombres de Argentina y Cuba respecto a la de otros países de Sur América es descripta por Bosetti. ${ }^{14}$ Las tasas globales del periodo fueron mayores en los hombres con una relación hombre mujer de aproximadamente 6,6 en Uruguay, 4,3 en Argentina y 2,2 en Cuba. El comportamiento en Argentina y Uruguay fue similar, con decremento temporal en los hombres e incremento en las mujeres. Las menores tasas para las mujeres de estos dos países junto con el gradual aumento observado sugieren que la gran «epidemia» de cáncer de pulmón entre las mujeres llegará en el futuro. Un comportamiento similar se observa en las últimas décadas en países desarrollados. ${ }^{13,31}$

La tasa global de mortalidad por cáncer de pulmón en Cuba no solo es la más alta del grupo sino que, en los últimos 10 años el PECA fue aún mayor que en la primera parte del periodo estudiado, lo que implica para Cuba un grave problema.

En el estudio de Bosetti y otros ${ }^{14}$ donde se comparan las tasas truncadas de mortalidad por diversos tumores en 10 países de Latinoamérica, de 1970 al 2000, se muestran comportamientos diversos en relación con el cáncer de mama. En ciertos países, caracterizados por tasas más bajas de mortalidad por este tumor, se describe un aumento temporal de las tasas, mientras que para otros, como Argentina y Cuba, se describe una estabilización aparente de las tasas, en las últimas tres décadas (no se hicieron cálculos del porcentaje de cambio anual).

En el presente trabajo, el cáncer de mama fue la primera causa de muerte por cáncer en las mujeres de Argentina y Uruguay y la segunda en Cuba. Las tasas estandarizadas de mortalidad por esta causa en el periodo 2001-2005 fueron mayores en Uruguay y Argentina y a partir de 1990 mostraron un decremento gradual menor del $1 \%$ anual para los dos países y cercano al $3 \%$ para Cuba aunque aquí la disminución no fue estadísticamente significativa. La diferencia con Cuba pudiera en parte estar relacionada con la menor incidencia que se muestra en este país, ${ }^{33,34}$ debido quizás a diferencias en relación a algunos factores relacionados con esta enfermedad como son, la protección que otorga la lactancia materna y los efectos negativos de la obesidad, el alto consumo de grasas y proteínas animales y el consumo de alcohol.

El tamizaje mamográfico organizado ha mostrado ser efectivo en reducir la mortalidad. ${ }^{35,36}$ Sin embargo, en estos países no existe este tipo de programas que cubran a toda la población en riesgo. Por tanto, la disminución de la mortalidad encontrada no puede adjudicarse a efectos de detección oportuna sino más bien a cambios en estilos de vida y fundamentalmente a mejoras en los tratamientos.

Una alta mortalidad por cáncer de estómago para ambos sexos, aunque siempre mayor en hombres que en mujeres, se localiza para algunos países de América Latina, entre ellos, Chile, Brasil, Costa Rica y Ecuador. ${ }^{14}$ La tasa estandarizada 
promedio anual del periodo 2001-2005 fue ligeramente mayor en Uruguay que en Cuba y Argentina y el comportamiento a partir de 1990 fue siempre en descenso gradual, con PECA ente 1,0 y $2,7 \%$. Este mismo comportamiento favorable de la mortalidad por cáncer de estómago ha sido encontrado en la mayoría de los países del mundo. ${ }^{12,14}$ Diversas son las causas que explican este comportamiento: mejor conservación de los alimentos (aunque en el período estudiado ya los cambios en el modo de conservarlos eran menores que para períodos anteriores), mejoras en la composición de la dieta, con mas consumo de frutas y verduras, mayor control de la infección por Helicobacter pylori. ${ }^{12}$

En Argentina, a partir de los años 90, la tendencia decreciente fue similar en las diversas regiones pero la mortalidad por cáncer de estómago en los hombres de la región patagónica fue considerablemente mayor que en el resto del país. Los autores sugieren la realización de estudios donde además de los factores conocidos que influyen en la ocurrencia del cáncer de estómago se tengan en cuenta factores genéticos y étnicos. ${ }^{37}$ También en Uruguay se observaron heterogeneidades geográficas entre diferentes áreas del país en cuanto a la mortalidad por esta causa siendo la disminución de las tasas menor en el área central del país. ${ }^{38}$

En los países desarrollados la incidencia y mortalidad por cáncer de ovario ha ido decreciendo en las últimas décadas como consecuencia, al menos en parte, de la introducción del uso de contraceptivos orales y los cambios en los patrones reproductivos. ${ }^{39} \mathrm{Al}$ inicio del período estudiado la tasa en los tres países fueron cercanas al 3/100 000 y durante el mismo no se observaron cambios significativos en su comportamiento. La mortalidad por tumores colorectales ocupó el tercer lugar excepto para las mujeres en Uruguay y Argentina donde ocupa el segundo. Llama la atención en Cuba que la tasas en las mujeres fue mayor que en los hombres. Esto mismo está descrito para Costa Rica y Ecuador sin encontrar una explicación satisfactoria, lo que merece la pena ser estudiado. ${ }^{40}$

En Argentina se encontró un descenso significativo de las tasas en ambos sexos que no llegó a ser del 1\%. Este descenso podría en parte ser explicado por cambios favorables en la dieta con mayores consumos de frutas, vegetales y cereales ricos en fibras y también con diagnósticos más tempranos y consecuentemente un mejoramiento de las posibilidades de curación, aunque no hay datos sobre esto, en el país, aún sin programas organizados de detecciones de estos tumores, ha aumentado la difusión de pruebas diagnósticas. ${ }^{37}$

Bosetti y otros ${ }^{40}$ describen no solo un rango variable de las tasas de mortalidad por tumores colorectales en los diversos países latinoamericanos sino también variaciones en el perfil de su comportamiento temporal, lo que sugiere que los cambios pueden deberse a modificaciones en la dieta a lo largo del tiempo.

Aún no son del todo conocidas las causas del cáncer de vesícula y vías biliares; un cáncer más frecuente en mujeres que en hombres. La asociación más conocida de este tumor es con los antecedentes de colelitiasis. Otros factores de riesgo incluyen infecciones crónicas del tracto biliar, hábito de fumar, dieta y obesidad. ${ }^{41,42}$ Altas tasas de incidencia y mortalidad por esta causa fueron descriptas en ciertas poblaciones de América Latina con alto porcentaje de ancestros indios, lo que sugiere cierta predisposición genética a este tumor. ${ }^{43}$

En Argentina y Uruguay las tasas en periodo 2001-05 fueron semejantes mientras que fueron menores las encontradas en Cuba. El comportamiento, en cambio, mostró divergencias. En Argentina se encontró a partir del 2001 un descenso gradual, sin cambios en la pendiente, siendo la velocidad de decrecimiento en mujeres casi el doble que en los hombres (2,0 \% vs. 1,2 \%). En Uruguay un 
descenso estadísticamente significativo solo se constató para las mujeres. En Cuba, las tasas en los hombres decrecieron gradualmente a un ritmo del 2,7 \% anual pero para las mujeres el descenso inicial del $3 \%$ anual se incrementó considerablemente aunque el PECA fue no significativo. Un descenso en las tasas de mortalidad por cáncer de vesícula fue descrito para varios países del mundo, entre ellos Costa Rica, lo que sugiere que la disminución puede ser como consecuencia de mejores modalidades diagnósticas y la prevención de cálculos biliares. ${ }^{44}$

En los tres países las tasas de mortalidad por cáncer de vejiga en los hombres fueron de más del doble que en mujeres. Puesto que esta forma parte del grupo de neoplasias tabaco-dependientes, su incidencia y mortalidad debería estar afectada por la prevalencia del hábito y por sus cambios en el tiempo. En este estudio, el PECA global del periodo $90-05$ para los hombres fue cercano a $1 \%$, pero el ritmo de decrecimiento mostró un cambio en el interior del periodo tanto en Argentina como en Uruguay. Del 2002 al 2005 en Argentina y de 1990 a 1993 en Uruguay, las tasas presentaron una tendencia al aumento, aunque no significativo. Esto no es consistente con lo visto en la mortalidad por cáncer de pulmón en los hombres, que en los mismos periodos tuvo una disminución. Solo en el Uruguay las tasas de mortalidad por cáncer de vejiga en las mujeres mostraron un cambio anual estadísticamente significativo, lo que está reflejado por un PECA cercano al $2 \%$ lo que pudiera estar indicando una mejoría en la calidad de certificación.

Un cambio favorable en las tasas de los hombres también fue descrito para diversos países europeos y adjudicado a una disminución de la prevalencia del hábito de fumar junto con una reducción en exposiciones ocupacionales. ${ }^{45}$

Como ponen de manifiesto en su estudio comparativo Bosetti y otros, ${ }^{14}$ la mortalidad por cáncer de útero en América Latina excede la encontrada para EE.UU. y Canadá. Argentina, Cuba y Uruguay comparten para ciertos tumores, como el de mama, perfiles de mortalidad similares a los países más desarrollados. Por otra parte, para otros tumores, como los del cuello de útero, aunque las TAE resultan más bajas que en otros países de América Latina, aún este tumor se encuentra presente en el patrón de mortalidad de estos tres países, tal como sucede en los menos desarrollados.

Al estudiar la mortalidad por tumores malignos del útero, es importante tener en cuenta que en una proporción variable de los certificados de defunción adjudicados a esta causa no se encuentra definido claramente si el tumor se hallaba localizado en el cuerpo o en el cuello del útero. La proporción de estos certificados codificados como debidos a tumores en el útero sin otra especificación (Útero NOS) no solo es variable entre los países sino también en un mismo país a lo largo del tiempo. Esta limitada especificación topográfica en parte de los certificados de defunción por cáncer de útero indica insuficiente entrenamiento de quienes completan los certificados además de un posible reflejo de un cierto atraso en el diagnóstico lo que lleva a tumores extensos donde es difícil distinguir el origen. ${ }^{46}$

En Uruguay, esa importante disminución de las tasas de Útero NOS (peca $7 \%$ ) no parece reflejarse en aumento en la mortalidad de cérvix. Pudiera ser porque la mortalidad por cáncer de cérvix se ve afectada por certificados de defunción que antes eran de útero NOS que ahora, al estar bien codificados pasan a cérvix.

En Argentina, analizando las tendencias de la mortalidad en las distintas localizaciones del útero, se ve que, para los tumores del cuello las tasas se mantuvieron prácticamente estables a lo largo del período, sin cambios significativos y lo mismo ocurrió de 1990 al 2000 para los tumores de localización indefinida dentro del útero. Contrariamente, las tasas correspondientes a tumores 
del cuerpo uterino disminuyeron casi el $3 \%$ al año. Esta situación, como fuera ya mencionada por Rocco ${ }^{47}$ debe asociarse más a mejoras en la confección de los certificados de defunción y no a una modificación en la situación epidemiológica real pues no hubo en el período grandes cambios en los tratamientos de este tumor que además no cuenta con estrategias de detección precoz que permitan disminuir su mortalidad.

La participación conjunta en REDEPICAN, financiada por el Programa CYTED (www.cyted.org), ha permitido la realización de este tipo de estudio que ayuda a evaluar las diferencias y similitudes que se presentan entre los países de América Latina. Como en el caso de Argentina, Cuba y Uruguay, que comparten algunos patrones de mortalidad semejantes a los de muchos países desarrollados, no obstante, se presentan ciertas diferencias. Mucho queda aún por investigar y de este modo dar respuesta a algunas interrogantes que aún están pendientes y quizás incorporar a otros países de la RED.

\section{Agradecimientos}

El siguiente trabajo agradece al proyecto de redes temáticas REDEPICAN (Red Iberoamericana de Epidemiología y Sistemas de información en cáncer) financiado por el Programa Iberoamericano de Ciencia y Tecnología para el Desarrollo (CYTED), quien ha facilitado el intercambio multidisciplinario de los profesionales de los diferentes países de la región. También damos las gracias a la Dirección Nacional de Estadísticas de la República de Cuba, a la Dirección de Estadísticas e Información de Salud del Ministerio de Salud y Ambiente de la nación Argentina y al Ministerio de Salud y la Comisión Honoraria de Lucha contra el Cáncer de Uruguay, por la disponibilidad de los datos de mortalidad por cáncer.

\section{REFERENCIAS BIBLIOGRÁFICAS}

1. CEPAL. Comisión Económica para América Latina y el Caribe. Conferencia regional intergubernamental sobre envejecimiento: Estrategia regional de implementación para América Latina y El Caribe del plan de acción internacional de Madrid sobre el envejecimiento. Santiago de Chile, 19-21 noviembre 2003[sitio en Internet]. [citado 13 Ago 2008]. Disponible en: http://www.eclac.cl/celade/noticias/paginas/1/13611/FINAL-DSC-1-Espanol.pdf

2. Lence Anta JJ, Camacho R. Cáncer y transición demográfica en América Latina y el Caribe. Rev Cubana Salud Pública [serie en Internet]. 2006 Sep [citado 10 Ago 2008 ];32(3): Disponible en: http://scielo.sld.cu/scielo.php?script=sci arttext\&pid=S0864$\underline{34662006000300010 \& \operatorname{lng}=\text { es\&nrm }=\text { iso }}$

3.Population Referente Bureau. 2006. World Population Data Sheet. United Nations. 2003 [sitio en Internet]. [citado 11 Ago 2008]. Disponible en: http://www.prb.org/pdf06/06WorldDataSheet.pdf

4. MINSAP/DNE. Serie de Anuarios Estadísticos de Salud. Ministerio de Salud Pública. Dirección Nacional de Estadísticas. La Habana, Cuba. 1990-2005 [sitio en Internet]. [citado 11 Ago 2008 ]. Disponible en: http://bvs.sld.cu/cgibin/wxis/anuario/?IsisScript=anuario/iah.xis\&base=anuario\&lang=e 
5. Dirección de Estadísticas e Información en Salud (DEIS). Indicadores

Seleccionados de Salud. República Argentina. Años 1980 a 1999. Serie 12 No.3, 2001[sitio en Internet]. [citado 15 Ago 2008]. Disponible en:

http://www.deis.gov.ar/publicaciones/Archivos/Serie12nro3.pdf

6. Dirección de Estadísticas e Información en Salud (DEIS). Indicadores

Seleccionados de Salud. República Argentina. Años 2000 a 2005. Serie 12 No.5, 2007 [sitio en Internet]. [citado 15 Ago 2008]. Disponible en:

http://www.deis.gov.ar/publicaciones/Archivos/Serie12nro5.pdf

7. Migliónico A. La mortalidad en Uruguay en el siglo XX. Montevideo: Ministerio de Salud Pública;2001.

8. Ministerio de Salud Pública-Uruguay. División Estadística sitio en Internet . citado 11 Ago 2008 . Disponible en: http://www.msp.gub.uy

9. World Health Organization. Global Infobase Cancer Country Profiles[sitio en Internet]. [citado 13 Ago 2008]. Disponible en:

http://www.who.int/infobase/cancer.aspx

10. Ferlay J, Bray F, Pisani P, Parkin DM. GLOBOCAN 2002. Cancer Incidence, Mortality and Prevalence Worldwide. IARC CancerBase No. 5, version 2.0. Lyon: IARCPress;2004.

11. Levi F, Lucchini F, Negri E, Boyle P, La Vecchia C. Mortality from major cancer sites in the European Union, 1955-1998. Ann Oncol. 2003;14:490-5.

12. Levi F, Lucchini F, Negri E, Boyle P, La Vecchia C. Cancer mortality in Europe, 1995-1999, and an overview of trends since 1960. Int J Cancer. 2004;110:155-69.

13. Jemal A, Siegel R, Ward E, Hao Y, Xu J, Murray T, et al. Cancer Statistics, 2008. CA Cancer J Clin. 2008;58:71-96.

14. Bosetti C, Malvezzi M, Chatenoud L, Negri E, Levi F, La Vecchia C. Trends in cancer mortality in the Americas, 1970-2000. Ann Oncol. 2005;16:489-511.

15. Matos E, Loria D, Zengarini N, Fernandez M, Guevel G, Marconi E, et al. Atlas de Mortalidad por Cancer en Argentina 1997-2001. Buenos Aires: Fundacion Bunge y Born [sitio en Internet]. 2003[citado 11 Ago 2008]. disponible en:

http://www.asarca.org.ar/archivos/AtlasMortalidadCancerArg97-01.pdf

16. Lence Anta JJ, Fernández Garrote LM. Tendencia de la incidencia de cáncer de laringe en Cuba, 1988-2003. Rev Cubana Salud Pública [serie en Internet]. Sep 2008 [citado 10 Ago 2008 ];34(3). Disponible en:

http://scielo.sld.cu/scielo.php?script=sci arttext\&pid=S0864-

34662008000300003\&lng=es\&nrm=iso

17. Becker RA. Lineamientos básicos para el análisis de la mortalidad. Programa Análisis de la Situación de Salud y sus Tendencias (HST) Oficina Sanitaria Panamericana (OPS) Pub. No. PNSP/92-15. Washington, D.C.: OPS;1992.

18. Kim HJ, Fay MP, Feuer EJ, Midthune DN. Permutation tests for joinpoint regression with applications to cancer rates. Stat Med. 2000;19:335-51. 
19. Percy C, Van Holten V, Muir C. ICD-O: International Classification of Diseases for Oncology. 2nd ed. Geneva: World Health Organization;1976.

20. Instituto Nacional de Estadísticas. Uruguay. Publicaciones en línea correspondientes a los Censos de 1985, 1996 y 2004[sitio en Internet]. [citado 11 Ago 2008]. Disponible en: http://www.msp.gub.uy/subcategorias 19 1.html

21. Boyle $P$, Parkin DM. Métodos estadísticos para los registros. En: Registros de Cáncer: Principios y Métodos. Cap.9. Lyon: IARCPress;1995.p.117-52. [Publicación científica No. 95].

22. Parkin DM, Whelan SL, Ferlay J, Teppo L, Thomas DB. editors. Cancer Incidence in Five Continents. Vol. VIII. Lyon: IARCPress;2002. [Publicación científica No. 155].

23. Kim HJ, Fay MP, Yu B, Barrett MJ, Feuer EJ. Comparability of Segmented Line Regression Models. Biometrics. 2004;60:1005-14.

24. Vives $A$, Valdivia G, Marshall G. Recent changes in prostate cancer mortality in Chile. Trends analysis from 1955 to 2001. Rev Med Chil. 2004;132(5):579-87.

25. Cayuela A, Rodríguez-Domínguez S, Vigil Martín E, Barrero Candau R. Cambios recientes en la mortalidad por cáncer de próstata en España: estudio de tendencias en el período 1991-2005. Actas Urol Esp[serie en Internet]. 2008[citado May 2009]32(2). Disponible en: http://scielo.isciii.es/pdf/aue/v32n2/v32n2a05.pdf

26. Vigil Martín E, Barrero Candau R. International trends in prostate-cancer mortality: the decrease is continuing and spreading. Cancer Causes Control. $2004 ; 15(3): 237-41$.

27. Galán Y, Guerra M, Fernández L, Camacho R. Incidence, mortality and survival from prostate cancer in Cuba, 1977-1999. Eur J Cancer Prevention.

2004;13(5):377-81.

28. Fernández L, Galán $Y$, Jiménez R, Gutiérrez A, Guerra M, Pereda $C$, et al. Sexual behaviour, history of sexually transmitted diseases and the risk of prostate cancer: a case-control study in Cuba. Int J Epidemiol. 2005;34:193-7.

29. Guha N, Boffetta P, Wünsch Filho V, Eluf Neto J, Shangina O, Zaridze D, et al. Oral health and risk of squamous cell carcinoma of the head and neck and esophagus: results of two multicentric case-control studies. Am J Epidemiol. 2007;166(10):1159-73.

30. Bogado ME, Araya FS, Alonso FT. Análisis de la mortalidad por cáncer en Chile (1990-2004). Acta Otorrinolaringol Esp. 2007;58 (8):336-40.

31. Bosetti C, Levi F, Ferlay J, Garavello W, Lucchini F, Bertuccio P, et al. Trends in oesophageal cancer incidence and mortality in Europe Istituto di Ricerche Farmacologiche "Mario Negri". Int J Cancer. 2008;122;1118-29.

32. Bosetti C, La Vecchia C. Cancer Mortality in Latin America: implications for prevention. Rev Panam Salud Publica. 2005;18(1):1-4.

33. Galan Y, Fernández L, Torres P, Guerra M, García M. Breast Cancer Risk in Cuba. Medicc Review. 2003; V(2-3). 
34. Torres P, Guerra M, Galán Y, García M, Lezcano M, Fernández L. Incidencia y mortalidad por cáncer en la mujer cubana. Trienio 2000-2002. Rev Cubana Med[serie en Internet]. 2007[citado May 2009];46(2). Disponible en: http://scielo.sld.cu/scielo.php?script=sci abstract\&pid=S003475232007000200007\&lng=es\&nrm=iso

35. Maxwell Parkin D, Fernández Garrote L. Statistics to Assess the Global Burden of Breast Cancer. The Breast Journal 2006;12 (Issue s1):S70-S80.

36.Shyyan R, Sener S, Anderson B, Fernández L, Hortobágyi G, Ibarra J et al. Guideline implementation for breast healthcare in low- and middle-income countries: Diagnosis resource allocation. Cancer. 2008;113 (S8):2257-68.

37. Atlas de Tendencias de Mortaliad por Cáncer Argentina 1980 - 2001. [Monografía en Internet]. [citado May 2009].Disponible en: http://www.asarca.org.ar/archivos/AtlasMortalidadCancerArg97-01.pdf

38. Barrios E, Álvaro L, Ronco LF, De Stéfani E, Vassallo JA. Tendencias de la mortalidad por cáncer en Uruguay 1953-1997. Rev Med Uruguay. 2002;18:167-74.

39. La Vecchia C. Epidemiology of ovarian cancer: a summary review. Eur J Cancer Prev. 2001;10:125-9.

40. Bosetti C, La Vecchia C. Cancer Mortality in Latin America: implications for prevention. Rev Panam Salud Publica. 2005;18(1):1-4.

41. Hariharan D, Saied A, Kocher HM. Analysis of mortality rates for gallbladder cancer across the world. Hariharan D, Saied A, Kocher HM. Analysis of mortality rates for gallbladder cancer across the world. HPB (Oxford) [serie en Internet]. 2008[citado May 2009];10(5). Disponible en:

http://www.ncbi.nlm.nih.gov/pmc/articles/PMC2575684/

42. C de Martel, M Plummer J Parsonnet, L-J van Doorn and S Franceschi. Helicobacter species in cancers of the gallbladder and extrahepatic biliary tract. British J Cancer. 2008.[25 November].

43. Ponce Lascano E, Miquel JF, Muñoz N, Herrero R, Ferreccio C, Witsuba I, ET AL. Epidemiology and Molecular pathology of Gallbader cancer. Cancer J Clin. $2001 ; 51: 349-64$.

44. Andia M, Gederlini GA, Ferreccio C. Cáncer de vesícula biliar: Tendencia y distribución del riesgo en Chile. Rev Méd Chile. 2006;134:565-74.

45. Ferlay J, Randi G, Bosetti C, Levi F, Negri E, Boyle P, Et Al. Declining mortality from bladder cancer in Europe. BJU International. 2008;1(1):11-9.

46. Arbyn M, Geys H. Trend of cervical cancer mortality in Belgium (1954-1994): tentative solution for the certification problem of unspecified uterine cancer. Int J Cancer. 2002;02:649-54.

47. Rocco D. Mortalidad por cáncer de útero en Argentina [sitio en Internet]. [citado 13 Ago 2008]. Disponible en:http://www.msal.gov.ar/htm/site/pdf/mortalidad.cancer.utero12.pdf 
*Argentina, Cuba y Uruguay son países miembros del proyecto de redes temáticas REDEPICAN ((Red Iberoamericana de Epidemiología y Sistemas de información en cáncer).

Instituciones que auspician el trabajo: REDEPICAN (Red Iberoamericana de Epidemiología y Sistemas de información en cáncer), Instituto Nacional de Oncología y Radiobiología y Escuela Nacional de Salud Pública.

Recibido: 28 de mayo de 2009.

Aprobado: 25 de enero de 2010.

Dora Loria. Departamento de Carcinogénesis Química y Ambiental, Área Investigación. Universidad de Buenos Aires, Instituto de Oncología "Ángel H. Roffo." Av. San Martin 5481, Buenos Aires 1417, Argentina.

Tel.: +54-11- 6568-6773; fax: + 54-11-4580-2811. E-mail: loriadora@yahoo.com 